\title{
PROPUOISERVIOE InFRminton
}

$\checkmark$ Electron Microscopy Sciences is proud to introduce the Oscillating Tissue Slicer. The most up to date, reasonably priced solution to sectioning fresh or fixed tissue without the need for embedding or freezing. A thermo electric refrigeration unit is available that connects to the main system. Electron Microscopy Sciences, PO Box 251, Fort Washington, PA 19034. Tel.: (215) 646-1566.

\section{$\checkmark$ EDAXINTERNATIONAL announces two revolutionary} energy dispersive $X$-ray microanalysis systems:

NX Series: With a fully integrated UNIXTM SPARC ${ }^{T M}$ station and Open Look ${ }^{T M}$ user interface, the new NX Series is designed to maximize user productivity and applications flexibility. A simple but powerful network can be built to allow an operator in the laboratory to collect data while others can simultaneously process data or generate reports.

DX Series: The world's first fully-integrated Microsoft ${ }^{M}$ Windows ${ }^{T M}$-based microanalyzer. Combining workstation performance with PC compatibility, plus an extensive range of options including digital imaging, mass storage devices and report generation, allows the new DX Series to offer the latest in user convenience and efficiency of operation.

EDAX INTERNATIONAL, 91 McKee Drive, Mahwah, NJ 07430. Tel.: (201)529-4880 $\checkmark$ DIATOME introduces the Cryo Family. With the addition of our Cryo Trimming Tool and Static Line lonizer, in combination with our Cryo Diamond Knives, it is now possible to achieve absolutely perfect ribbons of ultra thin frozen sections easily. The perfect match for all of your cryo ultramicrotomy needs. DIATOME, PO Box 125, Fort Washington, PA 19034. Tel.: (215)646-1478.

$\checkmark$ SEM-CLEAN is the most cost effective way to rid your SEM of oil contamination. Contact Ron Vane at XEI Scientific, 3124 Wessex Way, Redwood City, CA 94061. Tel.: (415)3690133.

$\checkmark$ Forensic Science Consulting in trace evidence, gunshot residue, electron microscopy, microanalytical techniques, image analysis and forensic anthropology. Competitive rates. Contact Max M. Houck at (817)377-4136 or (817)923-4999.

$\checkmark$ Scanning Electron Microscope Training: Customized training programs available to meet the specific needs of a laboratory - on your own equipment. Over twenty years of experience. Contact Robert Jemigan, SEMTRAINING, PO Box 800008, Sugar Hill, GA 30518. Tel:: (404)271-1857. 